\title{
Flexible or leaky attention in creative people? Distinct patterns of attention for different types of creative thinking
}

\author{
Darya Zabelina $^{1} \cdot$ Arielle Saporta $^{2} \cdot$ Mark Beeman $^{2}$
}

Published online: 2 November 2015

(C) Psychonomic Society, Inc. 2015

\begin{abstract}
Creativity has been putatively linked to distinct forms of attention, but which aspects of creativity and which components of attention remains unclear. Two experiments examined how divergent thinking and creative achievement relate to visual attention. In both experiments, participants identified target letters ( $\mathrm{S}$ or $\mathrm{H})$ within hierarchical stimuli (global letters made of local letters), after being cued to either the local or global level. In Experiment 1, participants identified the targets more quickly following valid cues $(80 \%$ of trials) than following invalid cues. However, this smaller validity effect was associated with higher divergent thinking, suggesting that divergent thinking was related to quicker overcoming of invalid cues, and thus to flexible attention. Creative achievement was unrelated to the validity effect. Experiment 2 examined whether divergent thinking (or creative achievement) is related to "leaky attention," so that when cued to one level of a stimulus, some information is still processed, or leaks in, from the non-cued level. In this case, the cued stimulus level always contained a target, and the non-cued level was congruent, neutral, or incongruent with the target. Divergent thinking did not relate to stimulus congruency. In contrast, high creative achievement was related to quicker responses to the congruent than to the incongruent stimuli, suggesting that real-world creative achievement is indeed associated with leaky attention, whereas standard laboratory tests of divergent thinking are not. Together, these results
\end{abstract}

Darya Zabelina

darya.zabelina@u.northwestern.edu

1 Medical Social Sciences (MSS), Northwestern University, 633 North Saint Clair St, Chicago, IL 60611, USA

2 Psychology Department, Northwestern University, Chicago, IL, USA elucidate distinct patterns of attention for different measures of creativity. Specifically, creative achievers may have leaky attention, as suggested by previous literature, whereas divergent thinkers have selective yet flexible attention.

Keywords Attentional flexibility · Cognitive control . Creativity $\cdot$ Divergent thinking $\cdot$ Creative achievement

Creativity, like many mental behaviors, requires attention. Prior evidence has suggested that creative people or creative acts are associated with distinct types of attention. However, several variant relations between creativity and attention have been posited, such as creativity relating to the broad attentional scope (Ansburg \& Hill, 2003), "leaky" attention, i.e., attention that allows "irrelevant" information to be noticed (Carson, Peterson, \& Higgins, 2003; Mendelsohn \& Griswold, 1964; Rawlings, 1985), broad conceptual scope (Rowe, Hirsh \& Anderson, 2007), flexible attention (Vartanian, Martindale, \& Kwiatkowski, 2007; Zabelina \& Robinson, 2010), and executive cognition, which relies heavily on the ability to focus attention (Nusbaum \& Silvia, 2011). These distinctions may arise because there are numerous forms, or measures, of creativity, such as successful performance on laboratory tests of divergent thinking, which assess the ability to find multiple solutions to a given problem within a limited amount of time, versus a measure of people's realworld creative achievements, assessed by a survey of people's creative achievements over their lifetime, as well as numerous forms of attention, with multiple components to each. Different measures of creativity may each emphasize different types or degrees of attention. Here we examine whether and how the two measures of creativity, divergent thinking and real-world creative achievement, are related to selective attention; that is, how people selectively attend to one stimulus (or 
element) among many, and how flexibly they switch between different foci of attention. Additionally, we investigate whether a diffused, or "leaky," attention (Fredrickson \& Branigan, 2005; Gasper \& Clore, 2002) provides a mechanism for flexible attention.

Creative cognition is often investigated in the context of divergent thinking. However, the correlations between divergent thinking and real-world creativity generally vary considerably, suggesting that they involve some unique processes (Runco \& Acar, 2012; Torrance, 1969; Zabelina, Condon, \& Beeman, 2014). Real-world creative achievements may indeed rely on the ability to think in a divergent manner, but may also reflect other factors, such as people's motivation, persistence, opportunity, resources, and personality. Additionally, the manner in which standard divergent thinking tests are administered and scored are often not identical to the environment in which real-world creative ideas are generated and produced. Unlike in the real-world, standard divergent thinking tests are administered in a laboratory with the instructions to find as many solutions as possible to a given problem within very constrained time limits (typically 2 or $3 \mathrm{~min}$ ). Thus successful performance on the divergent thinking test may require focus, generation, and inhibition of an already given response in order to be able to quickly move on to the next response.

An increasing body of research suggests that performance on divergent thinking tests involves top-down control of attention and cognition. Much of this evidence comes from latent variable studies showing effects of higher-order cognitive abilities, such as fluid intelligence (Beaty, Silvia, Nusbaum, Jauk, \& Benedek, 2014; Nusbaum \& Silvia, 2011), working memory capacity (Lee \& Therriault, 2013; Süß, Oberauer, Wittman, Wilhelm, \& Schulze, 2002), and verbal fluency (Benedek, Könen, \& Neubauer, 2012; Silvia, Beaty, \& Nusbaum, 2013). Such abilities are hypothesized to support thinking in a divergent manner by providing the executive control needed to guide memory retrieval and inhibit salient but unoriginal ideas (Beaty \& Silvia, 2012; Beaty et al., 2014; Benedek et al., 2012, 2014; Silvia, Beaty, Nusbaum, Eddington, \& Kwapil, 2014).

Behavioral evidence for the role of executive processes in performance on the divergent thinking tasks has already received support from electroencephalogram (EEG) and functional magnetic resonance imaging (fMRI) research. Several studies report task-related activation in brain regions associated with interference resolution, response selection, and cognitive control in the inferior frontal gyrus (IFG) and inferior parietal cortex (Abraham, Beudt, Ott, \& von Cramon, 2012; Benedek et al., 2014; Chrysikou \& Thompson-Schill, 2011; Fink et al., 2009; Fink \& Benedek, 2014).

Our prior work suggests that divergent thinking and realworld creative achievement have different associations with how people switch attention and process sensory information.
Specifically, creative achievement, but not divergent thinking, is associated with making more errors when switching levels of attention (from the broad to the narrow scope of attention, and vice versa) after remaining at a particular attentional level for some time (Zabelina \& Beeman, 2013), suggesting that creative achievement is related to attentional perseveration. We proposed that creative achievers' attention may be less focused, or "leaky." Whereas this leaky attention is beneficial for detecting alternative foci, leading to creative cognition, it may also demand that highly creative people exert more cognitive control in order to focus, and it takes time or effort to disengage this cognitive control, leading creative achievers to make more errors when switching levels of attention (Zabelina \& Beeman, 2013; also see Zabelina \& Robinson, 2010).

Indeed, neurophysiological evidence suggests that people with high real-world creative achievements have the reduced ability to filter out "irrelevant" sensory information, as assessed by the P50 event-related potential (ERP; Zabelina, O’Leary, Pornpattananangkul, Nusslock, \& Beeman, 2015). Additionally, they do it involuntarily, as this happens very early in the processing stream - only $50 \mathrm{~ms}$ after the onset of the sensory stimulus. This is akin to reduced latent inhibition, or a reduced ability to screen or inhibit from conscious awareness stimuli previously experienced as irrelevant, which has previously been linked to creative achievement (Carson et al., 2003). Creativity, as assessed by Mednick's (1962) Compound Remote Associates Tests (RAT; Mendelsohn \& Griswald, 1964; Russell, 1976) or by Wallach and Kogan (1965) battery of creativity tests (Rawlings, 1985) has also been associated with broad, or leaky attention.

In direct contrast to creative achievement, divergent thinking is associated with increased ability to filter out "irrelevant" sensory information, as assessed by the P50 ERP (Zabelina et al., 2015). Specifically, we find that high divergent thinkers are more likely to filter out sensory stimuli than their less divergent counterparts. Therefore, although shared attentional processes may exist between divergent thinking and creative achievement, they may also be associated with unique ways to use attention.

Here we examine whether and how divergent thinking and creative achievement are related to selective attention in a behavioral paradigm. Experiment 1 assessed people's ability to flexibly switch level of attention within hierarchical stimuli, following cues to one level or the other (e.g., switching attention from the local to the global level, or vice versa) on a trialto-trial basis. Experiment 2 assessed the "leakiness" or selectivity of each person's attention filter (generally defined, including the low-level non-conscious form of selection) to examine whether a leaky attention filter provides a mechanism for flexible attention in divergent thinkers and creative achievers. In both experiments, participants viewed hierarchical letter stimuli (global-local letters, Navon, 1977). On each trial prior to the stimuli, participants were cued about which 
level a target would likely occur, and speed of response to targets across different conditions indexed attention.

In Experiment 1, we examined the ability to allocate attention and shift it when necessary. Prior to each trial, participants were cued that a target letter ( $\mathrm{S}$ or $\mathrm{H}$ ) would appear at either the local or at the global level. Eighty percent of the trials were preceded by valid cues. Critically, $20 \%$ of the trials were preceded by invalid cues, e.g., the cue indicated the local level, but the local letters were not targets and the global configuration formed a target letter, or vice versa. We assessed each person's capacity for flexible attention by computing their validity effect: how much longer they took to respond to targets following invalid cues than targets following valid cues (see Lamb \& Yund, 2000; Posner, 1980). Given prior literature, we expected that people with higher divergent thinking scores would show a smaller validity effect, indexing more flexible attention. With regard to creative achievement, we expected to see no association between creative achievement and attentional flexibility if this type of attention is not linked with creative achievement. Alternatively, if creative achievement is linked with particularly flexible attention, people with higher creative achievements would exhibit a smaller validity effect.

\section{Experiment 1}

\section{Method}

\section{Participants and design}

Because of the novelty of this study, the power test could not be calculated to estimate sample size. Previous studies demonstrate that the ratio of validly to invalidly cued targets (i.e., cue validity) influences the magnitude of the validity effect (Jonides, 1980, 1983; Eriksen \& Yeh, 1985; Madden, 1992). Because ours was an individual difference study, we planned to include data from 135 participants.

Participants were 166 undergraduate students who received course credit or a US\$10 payment for participating. ${ }^{1}$ Written consent was obtained from all participants for a protocol approved by Northwestern University's Institutional Review Board. Each participant was tested individually in a $60-\mathrm{min}$ session. Data from 11 participants were excluded because they were outliers (data $>2.5 \mathrm{SDs}$ from mean) based on their error rates $(n=5)$, slow response times $(n=1)$, or validity effect $(n=$ 5). Thus analyses included data from 155 participants (66 men, 83 women, six unreported; average age $=19.30$ years,

\footnotetext{
${ }^{1}$ An additional nine people participated, but failed to perform either the divergent thinking or the attention task, e.g. not responding or responding less accurately than chance, and will not be discussed.
}

$S D=1.63$ ) who performed both the divergent thinking and the attention tasks; due to protocol variations, we obtained CAQ data from only 135 participants.

The study used an independent-measure design. The independent variable was the validity effect, and dependent variables were divergent thinking and creative achievement.

\section{Materials and procedure}

\section{Divergent thinking}

Divergent thinking was assessed by the Abbreviated Torrance Test for Adults (ATTA: Goff \& Torrance, 2002) - a shortened form of the Torrance Test of Creative Thinking (Torrance, 1974). The ATTA consists of three activities (3 min each), one involving verbal (written) responses (e.g., generating problems that may arise from being able to walk on air or fly without being in an airplane or a similar vehicle), and two involving figural responses (e.g., using incomplete figures to make pictures). Responses are scored for fluency (i.e., a count of the number of pertinent responses), and originality (i.e., the number of responses that are not typically produced, according to normative data); scores are summed across the three activities (Goff \& Torrance, 2002). The total divergent thinking score reflects a weighted score of fluency plus two times originality, to equally weight the two scores, since the average fluency score (13.9) was approximately double the average originality score (7.4), similar to the norms reported by the test developers (Goff \& Torrance, 2002; see Runco \& Acar, 2012 for suggestions on scoring divergent thinking tests). The average divergent thinking score was 28.79 (SD $=8.12$, range $10-55$ ). Forty-five participants completed the ATTA on a different day to the day they performed the attention task; the remaining participants completed both on the same day.

Creative achievement was assessed with the Creative Achievement Questionnaire (CAQ; Carson et al., 2005): Participants indicated their prior achievements in ten creative domains (architectural design, creative writing, culinary arts, dance, humor, inventions, music, scientific inquiry, theater and film, and visual arts). Questions in the Visual Arts domain, for example, range from "I have no training or recognized talent in this area (score of 0 )" to "My work has been critiqued in national publications (score of 7)." In the Scientific Discovery domain scores range from "I have no training or recognized ability in this field (score of 0 )" to "My work has been cited by other scientists in national publications (score of 7)." Domain scores were then summed to form a single index of creative achievement. The CAQ has test-retest reliability of $r=.81$, internal consistency reliability of alpha $=.96$, and shows predictive validity against artist ratings of a creative product, $r=.59$. Even though CAQ is a 
self-report measure, it has ecological validity in that it reflects actual creative behavior in the real world.

In addition to calculating the overall creative achievement scores, we also computed participants' creative achievement scores within artistic and scientific domains separately, following Carson and colleagues (2005). Artistic achievements included achievements within visual arts, music, dance, creative writing, theater and film, and humor domains; scientific achievements included achievements within inventions, scientific discovery, architecture, and culinary arts domains.

Three participants' CAQ scores exceeded 2.5 SDs greater than the mean, and were replaced with the next largest CAQ score in the sample to limit skewing. Mean CAQ score was $15.10(S D=13.21$, range $0-52)$, mean artistic achievement score was $12.66(S D=14.28$, range $0-52)$, and mean scientific achievement score was $2.12(S D=3.42$, range $0-21)$, similar to the norms reported by the CAQ developers (Carson et al., 2005).

\section{Academic tests scores}

Because factors related to intelligence, generally, likely influence scores on both the divergent thinking test (see Nusbaum \& Silvia, 2011) and the Creative Achievement Questionnaire (see Carson, Peterson, \& Higgins, 2005), we included academic test scores, which are heavily influenced by intelligence as assessed by the WAIS-III (Coyle \& Pillow, 2008), as factors in separate regression analyses predicting (a) divergent thinking, and (b) creative achievement. Participants $(N=125)$ provided their academic achievement test scores (Scholastic Assessment Test (SAT) or American College Testing (ACT); College Board, 2012; ACT Inc., 2014), which we converted into percentile scores based on the national statistics for all test-takers in $2012(M=97.69, S D=3.67$, range $68-100)$. In prior studies in our laboratory, self-reported scores were confirmed with actual scores through the admissions office, and the two correlated $r=.97$ (Wegbreit, Suzuki, Grabowecky, Kouniso, \& Beeman, 2012). Fourteen people did not provide their academic test scores (therefore degrees of freedom will be different when academic test scores are included in the analyses). The range of scores was very narrow, so this measure should be interpreted cautiously.

\section{Attentional flexibility task}

We adapted the Local-Global letter task (Navon, 1977) to optimally test for attentional flexibility. On each trial, participants indicated by a button press on a keyboard which target letter occurred, an "S" or an "H." Targets could occur at either the local or global level (global letters: $38 \mathrm{~mm} \times 25 \mathrm{~mm}$, local letters: $6 \mathrm{~mm} \times 4 \mathrm{~mm}$ ). Of eight total composite stimuli, four were global targets composed of local distractors (an $S$ made of $E$ 's, an $S$ made of $A$ 's, an $H$ made of $E$ 's, an $H$ made of $A$ 's), and four were local targets forming a global distractor (an $E$ made of $S$ 's, an $E$ made of $H$ 's, an $A$ made of $S$ 's, an $A$ made of $H$ 's). Each vertical line making up a global letter was formed from five and each horizontal line was formed from four closely spaced local letters. Stimuli were pseudo-randomized, and presented centrally one at a time. The stimuli were adapted from a prior experiment, and were designed so that global and local stimuli elicit approximately equal response speed and accuracy (Bultitude, Rafal, \& List, 2009).

Prior to each trial, a centrally presented cue indicated at which level the target was likely to occur. Critically, $80 \%$ of the trials were preceded by a valid cue, and $20 \%$ of the trials were preceded by an invalid cue (i.e., following a local cue a target appeared only at the global level, or vice versa). Prior studies indicate that the ratio of validly to invalidly cued targets (i.e., cue validity) influences attentional allocation with high cue validities increasing the magnitude of the validity effect (Jonides, 1980, 1983; Eriksen \& Yeh, 1985; Madden, 1992). The global cue was a square outline the size of the global stimuli $(2.1 \mathrm{~cm} \times 2.1 \mathrm{~cm})$, and the local cue was a "+ " sign the size of the local stimuli $(0.4 \mathrm{~cm} \times 0.4 \mathrm{~cm}$; Fig. 1). Participants were instructed that each target letter would be preceded by a cue, and that on most trials the cue would indicate at which level a target would appear. Participants used a chin rest while performing the task to ensure similar distance between the participant and the computer display for all participants $(60 \mathrm{~cm})$. The global letters subtended a visual angle of $1.5^{\circ}$ vertically $\times 1$ degree horizontally, and the local letters $.25 \times .15$ degrees.

On each trial, participants were first presented with a cue in the center of the screen for $1,500 \mathrm{~ms}$, followed by a blank screen for $500 \mathrm{~ms}$. (These parameters were identified through pilot testing to allow participants to make best use of the cues in this paradigm.) Then, the composite stimulus appeared in random order for 2,000 ms, and participants pressed an "S" key if the stimulus contained the letter " $\mathrm{S}$ " or an " $\mathrm{H}$ " key if the stimulus contained the letter "H." Another blank screen was then presented for 1,000 ms. Participants were asked to respond as quickly and accurately as possible. After 16 practice trials, 136 trials were presented in pseudo-random order.

Our primary dependent variable was the validity effect, how much more slowly people responded following invalid cues than following valid cues (response time (RT) on invalid trials minus RT on valid trials, correct trials only).

Participants completed the attentional flexibility task, followed by the divergent thinking task (Goff \& Torrance, 2002). Participants who received course credit completed the Creative Achievement Questionnaire (Carson et al., 2005) as part of the class requirement on the first day of the class; participants who received payment for participation completed the Creative Achievement Questionnaire at the end of the testing session. Twenty participants did not complete the CAQ. 


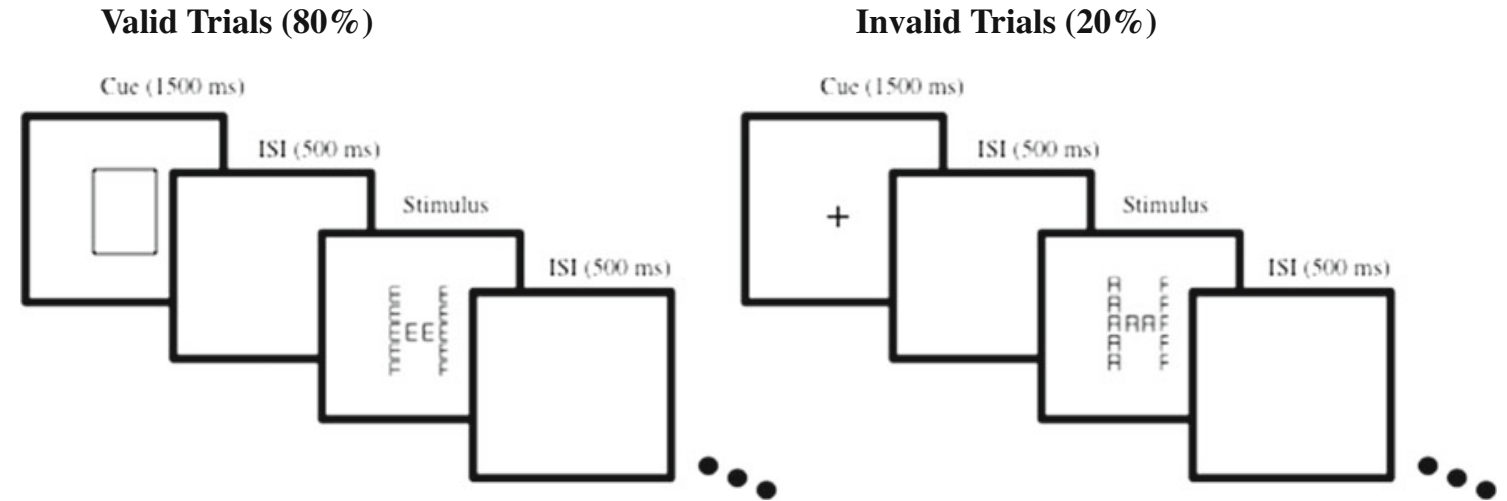

Fig. 1 An example of a valid and an invalid trial in Experiment 1. Participants are asked to identify whether there is an " $\mathrm{S}$ " or an " $\mathrm{H}$ " present on the screen. Each target is preceded by either a global cue

\section{Results}

On the attention task, overall participants responded to targets more quickly following valid cues $(M=720 \mathrm{~ms}, S D=107)$ than following invalid cues $(M=753 \mathrm{~ms}, S D=126)$, pairedsample $t(153)=6.33, p<.0001, d=1.02$, thus demonstrating a reliable $33-\mathrm{ms}$ validity effect, on average. The validity effect was slightly reduced as the task went on $(34 \mathrm{~ms}$ in the first half of the trials, $23 \mathrm{~ms}$ in the second half of the trials), pairedsample $t(154)=1.74, p=.08, d=.28$.

Participants responded slightly more quickly to local $(M=$ $721 \mathrm{~ms}, S D=109)$ than to global targets $(M=732 \mathrm{~ms}, S D=$ 112), paired-sample $t(153)=2.79, p<.01, d=.45$, but this did not interact with validity, as participants produced similar validity effects for both global $(30.78 \mathrm{~ms})$ and local $(36.88 \mathrm{~ms})$ targets, $p>.36$.

\section{Divergent thinking}

Our primary hypothesis was that people with higher divergent thinking scores (as measured by the ATTA) would exhibit flexible attention, indexed by the ability to overcome invalid cues. As predicted, higher divergent thinking scores were associated with smaller validity effects, $r(152)=-.23, p=.004^{2}$ (Fig. 2).

Linear regression confirmed that the validity effect remained a significant predictor of divergent thinking even after controlling for academic test scores and the overall response latency on the attention task (Table 1).

Confirming the stability of individual differences, the correlations between divergent thinking and the validity effect did not differ whether the ATTA was administered on the same

\footnotetext{
$\overline{2}$ The correlation remains reliable if log-transformed RTs are used, $r(152)$ $=-.25, p=.002$.
}

(large square) or a local cue (small plus sign). Eighty percent of the cues are valid, and $20 \%$ of the cues are invalid. Trials are presented in pseudo-random order

day $(r(108)=-.23, \mathrm{p}=.01)$ or on a different day $(r(43)=-.33$, $p<.03$ ), Fisher $r$-to- $z=.60, p=.55$.

Divergent thinking was unrelated to overall latency, or latency to identify global or local targets, $p \mathrm{~s}>.10$.

\section{Creative achievement}

Divergent thinking and real-world creative achievement were weakly associated, $r(135)=.17, p=.05$, indicating a slight relation between the two constructs. Yet, unlike divergent thinking, creative achievement was not related to the validity effect, in a direct correlation, $r(135)=.01, p=.96$, or in a linear regression controlling for general intelligence and overall latency to identify targets $(p>.78){ }^{3}$ Similarly, there was no association between the validity effect and creative achievements in the arts, $r(135)=-.05, p>.63$, or creative achievements in the science domains, $r(135)=.05, p>.63$. Creative achievement was not associated with overall latency, or latency to identify global or local targets, $p \mathrm{~s}>.95$.

\section{Error rates}

Participants made errors on $2.55 \%(S D=2.32)$ of the trials, and made more errors on trials with invalid cues $(M=3.65 \%, S D=$ 4.57) than on trials with valid cues $(M=2.29 \%, S D=2.07)$, paired-sample $t(154)=3.26, p=.001, d=.53$, and more errors on global $(M=3.01 \%, S D=2.94)$ than on local targets $(M=$ $2.33 \%, S D=2.55)$, paired-sample $t(154)=2.87, p=.005, d=$ .46. Note that the standard deviations are quite large because the error rate distribution was positively skewed, i.e., most

\footnotetext{
${ }^{3}$ Results maintained when two 2.5 SDs above the mean CAQ scores were used, rather than replaced with the next largest CAQ score. In these analyses correlation between divergent thinking and creative achievement was $r(133)=.15, p=.09$; correlation between creative achievement and congruency effect was $r(135)=-.01, p=.94$.
} 


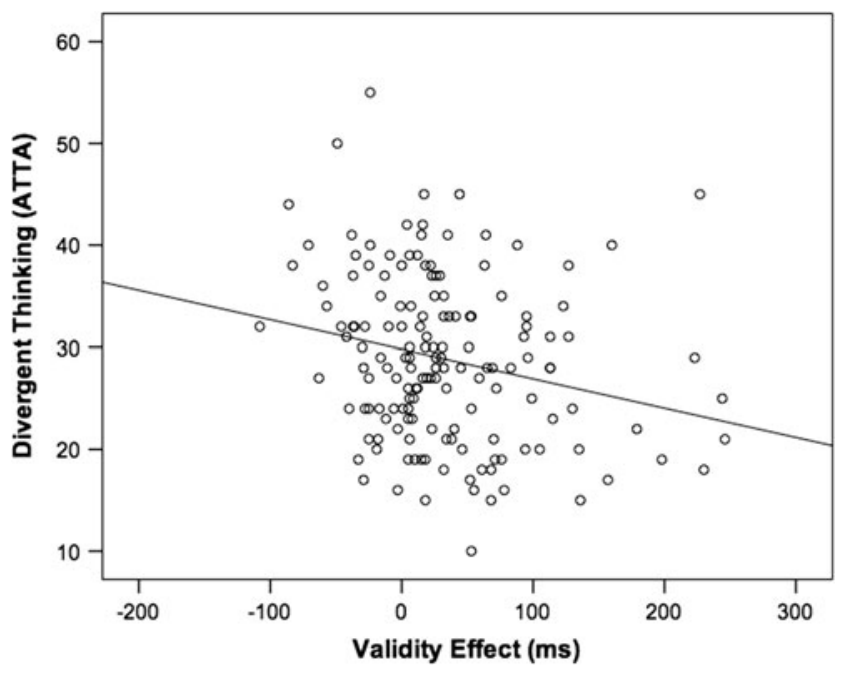

Fig. 2 A Pearson correlation between divergent thinking and validity effect (response time (RT) on invalid trials minus RT on valid trials), demonstrating that people with higher divergent thinking scores have more flexible attention

participants made zero or only a few errors, while a small number of participants made more than a few errors.

Neither divergent thinking, nor creative achievement were associated with overall error rates, or errors on valid and invalid trials, or global and local targets (all $p \mathrm{~s}>.13$ ).

\section{Discussion}

In Experiment 1, people with higher divergent thinking scores were better at overcoming invalid cues, even when controlling for academic test scores or overall response latency. Therefore, higher divergent thinkers were more flexible in their attention. In contrast, real-world creative achievement was not associated with the size of the validity effect, demonstrating that attentional flexibility, as measured here, was specific to divergent thinking.

There are several possible mechanisms for why divergent thinking was associated with better overcoming of invalid cues. One possible mechanism is a leaky attention filter. Specifically, when people attend to one level, information from the other level may still "leak in," allowing relatively easy identification of non-cued targets after invalid cues. Some prior evidence suggests that certain forms of creativity may be associated with the leaky attention filter (Ansburg \& Hill, 2003; Carson et al., 2003; Neçka, 1999; Rowe et al., 2007). Previously we found that creative achievement, but not divergent thinking, is associated with the reduced ability to filter our "irrelevant" sensory information (Zabelina et al., 2015). Experiment 2 examined whether attentional flexibility associated with divergent thinking is supported by leaky attention.

\section{Experiment 2}

Experiment 2 tested whether divergent thinking or creative achievement are related to a leaky attention filter, which would be manifested in the size of the congruency effect, i.e., how selective people are when cued to selectively attend to either the local or global letter stimuli. Participants were again cued to a stimulus level, but unlike in Experiment 1, the cued level always contained a target (either $\mathrm{S}$ or $\mathrm{H}$ ). However, the stimulus at the other level was congruent, incongruent, or neutral with the target. Thus congruency, not validity, was manipulated, as the cue was always valid. Specifically, there was always a target at the cued level of the stimulus, but we varied whether the other level contained congruent, neutral, or incongruent target information. For instance, when cued to the local level participants could see a local S; on congruent trials the global configuration formed the same letter (large $S$ ); on incongruent trials, the global letter was the other target (large $\mathrm{H}$ ); and on neutral trials the global letter was a non-target letter (A's or E's). The congruency effect - how much faster people respond on congruent than on incongruent trials - is an index of how selective the attention filter is. (Because the neutral level contained lessoften-seen letters, it may not be a good baseline for contrasting costs and benefits.) If people are perfectly good at using the cues to attend to the target level, the congruency of the distractor level will have no effect. On the other hand, if people have leaky filters, they will respond more slowly on incongruent trials. Thus, if divergent thinking in Experiment 1 was associated with better overcoming of invalid cues due to leaky attention, then high divergent thinking should also be associated with a large congruency effect in Experiment 2. In contrast, if better overcoming of invalid cues was associated with better overcoming of invalid cues not due to leaky attention in Experiment 1, then in Experiment 2 divergent thinking should not be related to the congruency effect.

Likewise, if real-world creative achievement is associated with leaky attention, then people with higher creative achievements should show larger congruency effects in Experiment 2.

Table 1 Divergent thinking as a function of validity effect, academic test scores, and overall speed on the attention task

\begin{tabular}{llllll}
\hline & $B$ & $S E B$ & $\beta$ & $t$ & $p$ \\
\hline Validity effect & -.02 & .01 & -.18 & 2.05 & .04 \\
Academic test scores & .30 & .19 & .14 & 1.58 & .12 \\
Response time on attention task & -.01 & .01 & -.11 & 1.20 & .24 \\
\hline
\end{tabular}




\section{Method}

\section{Participants and design}

As in Experiment 1, the power test could not be calculated because of the novelty of this study. Previous studies have demonstrated presence of a congruency effect in 16 individuals (Hedden $\&$ Gabrieli, 2011). Because of the individual difference nature of our study, we planned to include data from 96 participants.

One hundred and two undergraduate students (male/female $=46 / 53$, three unidentified, average age $=18.77$ years, $S D=$ 1.20) participated in the study for course credit or payment. Each participant gave informed consent prior to participating, and was tested individually in a 60-min session. Data from six participants were excluded based on large $(>2.5 S D$ s from mean) error rates $(n=4)$, long response times $(n=1)$, or an unusually large congruency effect $(n=1)$.

The study used an independent-measure design. Independent variable was the congruency effect, and dependent variables were divergent thinking and creative achievement.

\section{Materials and procedure}

\section{Divergent thinking}

Divergent thinking was assessed with the Abbreviate Torrance Test for Adults (ATTA:

Goff \& Torrance, 2002) with the same instructions and scoring procedure as in Experiment 1. Mean divergent thinking score was $28.31(S D=9.22$, range 7-60). Thirty participants performed an alternate version of the ATTA task. One participant did not follow test instructions, and was not included in the analyses.

The Creative Achievement Questionnaire (CAQ: Carson et al., 2005): We assessed participant's real-world creative achievements with the CAQ. In addition to calculating the overall creative achievement scores, we also computed participants' creative achievement scores within artistic and scientific domains separately. Mean CAQ score was $14.30(S D=12.24$, range 0 $45)$, mean creative achievement score in the artistic domain was $11.64(S D=11.02$, range $0-45)$, and mean creative achievement score in the science domain was $2.64(S D=3.76$, range $0-19)$.

Academic tests scores Participants $(N=83)$ provided their SAT or ACT scores, which were then converted into percentile scores based on the national statistics for all test-takers in 2012 $(M=96.54, S D=3.99$, range 79-100).

The Congruency Task directly examined individual differences in the leakiness or selectivity of attention. The stimuli were the same local-global letters (Navon, 1977) as in Experiment 1. Participants were cued to a stimulus level, which now always contained a target (either $\mathrm{S}$ or $\mathrm{H}$ ); but information at the other level was either congruent (33\%), incongruent (33\%), or neutral (34\%) with the target (e.g., when cued to the local level they saw a global $\mathrm{S}$, and it was comprised of either small $S$ 's, small $H$ 's, or small $E$ 's, respectively; Fig. 3).

As in Experiment 1, on each trial, participants saw a cue (always valid) in the center of the screen for $1,500 \mathrm{~ms}$, followed by a blank screen for $500 \mathrm{~ms}$. Then, one of 12 composite stimuli was randomly presented for $2,000 \mathrm{~ms}$, and participants were asked to use a keyboard to press an "S" if the cued level contained the letter $\mathrm{S}$, or an " $\mathrm{H}$ " if the cued level contained the letter H. Another blank screen followed for 1,000 ms. Participants were asked to respond quickly and accurately. Two of the composite letters were congruent stimuli (an $S$ made up of $S$ 's and an $H$ made up of $H$ 's), two were incongruent stimuli (an $S$ made up of $H$ 's and an $H$ made up of $S$ 's), and eight were neutral stimuli (an $S$ made of $E$ 's, an $S$ made of $A$ 's, an $H$ made of $E$ 's, an $H$ made of $A$ 's, an $E$ made of $S$ 's, an $E$ made of $H$ 's, an $A$ made of $S$ 's, an $A$ made of $H$ 's).

Overall, participants completed 166 trials, after 20 unanalyzed practice trials. Our primary interest was whether the congruency effect (RT on incongruent trials minus RT on congruent trials; correct trials only) varied with divergent thinking and creative achievement.

\section{Results}

Participants responded more quickly to congruent targets ( $M=$ $714 \mathrm{~ms}, S D=126)$ than to incongruent targets $(M=783 \mathrm{~ms}$, $S D=134)$, paired-sample $t(95)=-11.50, p<.001, d=.53$, demonstrating a reliable $69 \mathrm{~ms}$ congruency effect, on average. Participants responded with equal speed to global and local targets, $p>.54$, and had equal size congruency effect on the global and local targets, $p>.19$.

\section{Divergent thinking}

The goal of Experiment 2 was to examine whether creativity, by either measure, was related to a leaky attention filter. Specifically, if high divergent thinkers in Experiment 1 were flexible at overcoming invalid cues because information from the other stimulus level leaked in, then divergent thinking should also relate to the size of the congruency effect in Experiment 2.

In contrast with the leaky filter hypothesis, divergent thinking did not relate to the congruency effect in a direct correlation, $r(93)=.13, p=.22$, nor in a linear regression controlling for general intelligence and overall latency to identify targets on the Congruency task $(p>.37)$. Thus the hypothesis that divergent thinkers have leaky attention was not supported, suggesting that a different mechanism was in play by which divergent thinkers were better at overcoming invalid cues in Experiment 1. 
Congruent Trial

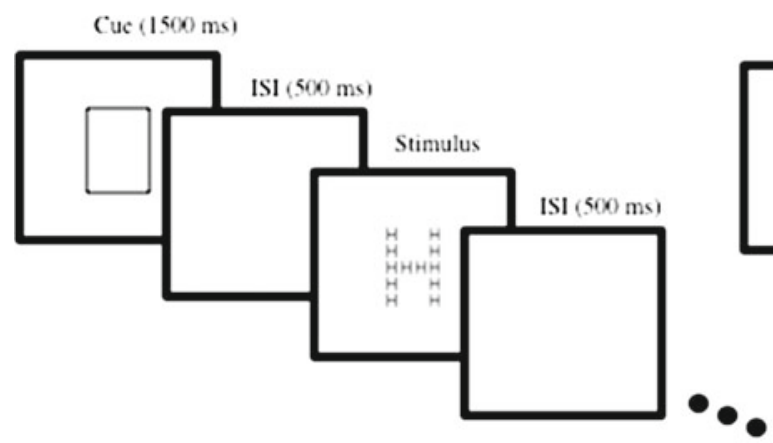

Fig. 3 An example of a congruent and incongruent trial in Experiment 2. Participants are asked to identify whether there is an "S" or an " $\mathrm{H}$ " present on the screen. Each target is preceded by either a global cue (large square)

Divergent thinking was not associated with overall latency, or latency to identify global or local targets, $p \mathrm{~s}>.11$.

\section{Creative achievement}

Divergent thinking and creative achievement were marginally associated in this sample, $r(93)=.17, p=.10$.

Unlike divergent thinking, real-world creative achievement was reliably associated with the congruency effect, $r(94)=$ $.22, p<.03^{4}$ (Fig. 4). Considering artistic and scientific creative achievements separately, we found that people with creative achievements in the arts had reliably larger congruency effect, $r(94)=.23, p=.04$, but creative achievements in the sciences were unrelated to the size of the congruency effect, $r(94)=-.04, p>.74$. Thus it appears that only people with creative achievements in the artistic domains have leaky attention.

This effect persisted when controlling for general intelligence and overall latency of responses within a linear regression (Table 2).

Creative achievement was unrelated to overall latency, or latency to identify global or local targets, $p \mathrm{~s}>.33$.

\section{Error rates}

Participants made errors on $4.80 \%(S D=3.18)$ of the trials, and made more errors on incongruent $(M=9.33 \%, S D=7.11)$ compared to congruent trials $(M=1.74 \%, S D=2.03)$, pairedsample $t(95)=10.81, p<.001, d=2.01$, and more errors on global $(M=5.58 \%, S D=4.00)$ compared to local targets $(M=$ $4.01 \%, S D=3.65)$, paired-sample $t(95)=3.83, p<.001, d=$ .96. As in Experiment 1, standard deviations are large because the error rate distribution was positively skewed.

\footnotetext{
$\overline{{ }^{4}}$ The correlation remains reliable if log-transformed RTs are used, $r(94)=$ $.20, p=.047$.
}

Incongruent Trial

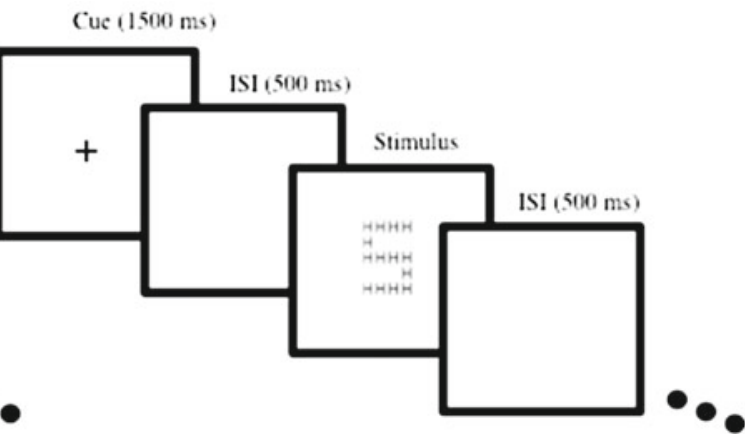

or a local cue (small plus sign). Cues are $100 \%$ valid. Trials are presented in pseudo-random order

Neither divergent thinking nor creative achievement were associated with overall error rates, or errors on congruent and incongruent trials, or global and local targets, $p \mathrm{~s}>.10$.

\section{General discussion}

The purpose of the investigation was to examine whether, and how, attentional flexibility is associated with creativity, as indexed by divergent thinking and creative achievement. Results from Experiments 1 and 2 demonstrate that different forms of attention relate to different measures of creativity. Specifically, we reveal a novel dichotomy in attention employed by divergent thinkers versus attention utilized by creative achievers.

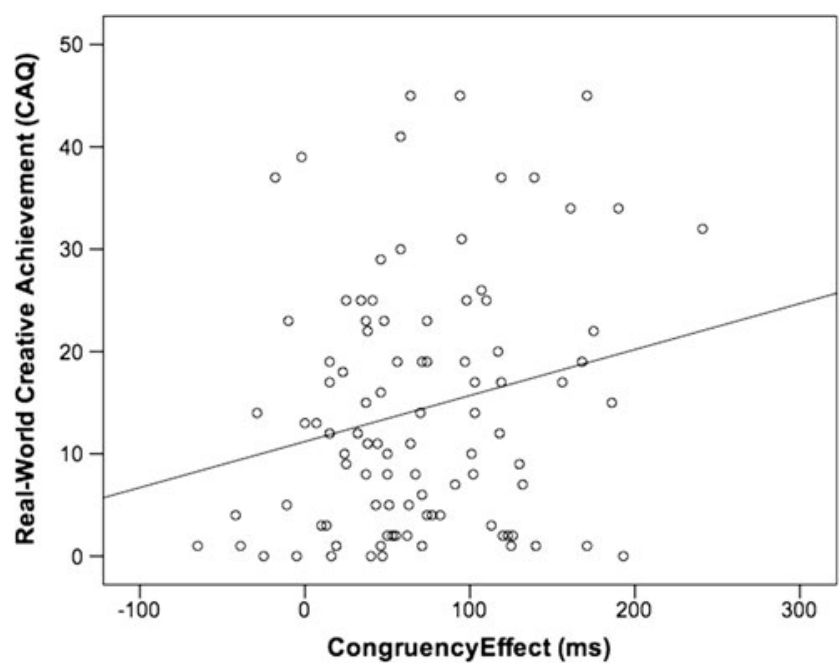

Fig. 4 A Pearson correlation between creative achievement and congruency effect (response time (RT) on incongruent trials minus RT on congruent trials), demonstrating that people with higher real-world creative achievements have more "leaky" attention 
Table 2 Creative achievement as a function of congruency effect, academic test scores, and overall speed on the attention task

\begin{tabular}{llllll}
\hline & $B$ & $S E B$ & $\beta$ & $t$ & $p$ \\
\hline Congruency effect & .07 & .02 & .32 & 3.04 & .003 \\
Academic test scores & .29 & .33 & .09 & .87 & .39 \\
Response time on attention task & -.01 & .01 & -.06 & -.57 & .57 \\
\hline
\end{tabular}

\section{Divergent thinking and selective attention filters}

Experiment 1 demonstrated that people with higher divergent thinking scores were less affected by invalid cues, even when accounting for general intelligence and overall latency of responses. Thus, divergent thinking was related to flexible attention, at least by this measure (also see Vartanian, 2009; Vartanian et al., 2007).

One possible mechanism by which divergent thinkers could overcome invalid cues is by having leaky attention filters, so that even when attention was cued to one level of a stimulus, information from the other level leaked in, easing the cost of switching attention after invalid cues. Experiment 2 revealed that divergent thinking was not associated with leaky attention. Divergent thinking was not related to either interference or facilitation from stimuli at the uncued level. This finding rules out leaky attention as the explanation for flexible attention (i.e., overcoming invalid cues) displayed by divergent thinkers in Experiment 1. Therefore an alternative mechanism may be responsible for divergent thinkers' flexible attention in Experiment 1. It is possible that a rather selective attention filter is the mechanism for the flexible attention in divergent thinkers. In this view, high divergent thinkers, when cued, focus their attention as sharply as (or sharper than) low divergent thinkers. Thus, high divergent thinkers, when necessary, are adept at rapidly processing information at the cued level, rapidly disengaging from it, and/or rapidly shifting to the other level. In other words, even though they are highly selective at attending to cued information, their attention capture is not as strong or as lasting. This is analogous to an effect found in the working memory literature, in which both high and low working memory capacity people exhibit attentional capture by distractors, but high capacity individuals recover more quickly than their low capacity counterparts, as indicated by behavioral and ERP findings (Fukuda \& Vogel, 2011).

Although the present experiments did not test this directly, such a mechanism is compatible with our recent data showing that divergent thinking is linked with an increased ability to filter out "irrelevant" sensory information (Zabelina et al., 2015). Considering the manner in which divergent thinking tests are typically administered (limited time and emphasis on the number of responses), selective attention would appear to facilitate successful performance on divergent thinking tests.

\section{Creative achievement and leaky attention filters}

Although there may exist shared attentional processes between divergent thinking and creative achievement, the two measures appear to be associated with unique ways to use attention. Experiment 2 provided evidence that, in contrast with divergent thinking, real-world creative achievement is associated with leaky attention. The more real-world creative achievements participants reported, the more they showed interference from the uncued level on incongruent trials (or facilitation on congruent trials). Specifically, when cued to a stimulus level, which always contained a target (either S or $\mathrm{H}$ ), real-world creative achievers responded more quickly if stimulus information at the other level was congruent, and more slowly if information at the other level was incongruent, with the intended response. Thus, information from the uncued level leaked in and affected the responses of people with high creative achievements more than it leaked in for people with fewer creative achievements. This was only true for people with artistic, rather than scientific creative achievements.

Prior empirical investigations have suggested a link between creative achievement and a leaky attentional filter (Carson et al., 2003; Zabelina et al., 2015), which may facilitate a wide focus on the weakly activated stimuli, leading to unusual and creative associations. This is akin to the remote association theory, which describes creativity as the ability to utilize non-dominant remote associations of problem elements in order to discover nonobvious solutions to a problem (Mednick, 1962). Thus, leaky attention may facilitate access to remote associations, and lead to a creative thought.

Creative achievement was not associated with the size of the validity effect in Experiment 1. Thus, a leaky filter does not appear to help in this task. It appears that any potential benefit a leaky filter could provide in the validity task was negated by other factors. Prior work with another version of the local-global task may point to such a mechanism (Zabelina $\&$ Beeman, 2013). Again, participants had to identify either an $\mathrm{S}$ or an $\mathrm{H}$ in hierarchical letter stimuli, but no cues were presented. Instead, the target occurred at the same level for five to nine sequential trials, before switching to the other level again, without any cues. High real-world creative achievers made more errors when the level switched, demonstrating an increased cost of switching attention (Zabelina \& Beeman, 2013). One possible reason creative achievers experienced this unwanted persistence is that, because they have leaky attention filters, they rely more heavily on cognitive control to maintain their focus of attention, and cognitive control that shields processing from distractions also makes switching more difficult (Dreisbach \& Goschke, 2004). Similarly, results from the present investigation indicate that high creative achievers have leaky attention filters (Experiment 2), but they cannot use that to help identify stimuli following invalid cues 
(Experiment 1). In the validity task, compared to people with lower creative achievement, high creative achievers may rely more on cognitive control, which prevents them from quickly shifting to the other stimulus level. In contrast, in the congruency paradigm of Experiment 2, they don't need to shift (a target was always present at the cued level), so the leaky filter simply allows information from the non-cued level to influence their response, without any switching cost.

Although our attention tasks rely heavily on visual attention, we would expect to see similar effects in tasks relying more on semantic processing and/or other sense modalities. Indeed, prior work shows that similar conclusions can be drawn from auditory modality (Zabelina et al., 2015), and semantic modality (Rowe et al., 2007). However, the fact that we find associations between flexible attention and divergent thinking as well as leaky attention and creative achievement at perceptual level is especially interesting, and suggests that these relations hold across modalities, signaling a general mechanism in divergent thinkers and creative achievers.

Although the ATTA and CAQ are accepted measures, their use leads to certain limitations in terms of the conclusions that can be drawn. The CAQ is a well-established measure, with high predictive validity against artist ratings of a creative product, and high convergent validity with other measures of creative potential (Carson et al., 2005), but it is quite broad, and encompasses more than just creative thinking. Moreover, some creative acts that are important to an individual may not register on the CAQ. In contrast, the ATTA is a more narrowly defined performance measure that theoretically contributes in part to creativity, and there is some evidence of the association between the ATTA and CAQ, though it is weak in our studies. Thus interpretations of the current work need to bare these caveats in mind when interpreting the results.

Finally, our sample consisted of young psychology students, therefore creative achievements in this sample may not entirely represent the overall population, especially in the scientific domains (the range of the CAQ scores in the science domains was quite limited compare to the scores in the arts domains). Future studies will need to explore how attention functions in creative professionals in various creative fields.

\section{Overall conclusion}

Although divergent thinking and creative achievement are both measures of creativity, and are weakly related, they appear to be associated with unique ways to use attention. In the present study, divergent thinking was associated with flexible attention, the mechanism of which does not appear to be leaky attention. We suggest that selective focus and inhibition components of attention may facilitate successful performance on the current measures of divergent thinking, given their emphasis on the number and the speed of responses. Therefore some components of divergent thinking tests may emphasize focus and inhibition, rather than the ability to think in a divergent manner. Further investigations are warranted to test this proposal.

Real-world creative achievement, on the other hand, appears to relate to leaky attention. If high creative achievers' attention is truly leaky, and they underperform low achievers on the standard laboratory tests of selective attention, then what enables them to persist with an idea or behavior that ultimately leads to a new musical composition, or a highly original painting? One possibility is that high real-world creative achievers have learned to exert more cognitive control in order to maintain their attention when needed. Whereas leaky attention is beneficial for detecting alternative foci and switches quickly, cognitive control takes longer to switch. Future investigations are needed here as well.

In summary, Experiments 1 and 2 demonstrate that different attention mechanisms independently relate to divergent thinking and to real-world creative achievement. These results refine the long-standing contention that "creative people" are generally more distractible or have leaky attention. Instead, we demonstrate that different measures of creativity each emphasize different types of attention. Specifically, creative achievement may be associated with leaky attention while divergent thinking is linked with selective yet flexible attention.

Acknowledgments This research was supported by the NIH grant T32047987, grant RFP-15-04 from the Imagination Institute, funded by the John Templeton Foundation to DLZ, and John Templeton Foundation grant 24467 to $\mathrm{MB}$.

\section{References}

Abraham, A., Beudt, S., Ott, D. V. M., \& von Cramon, D. R. (2012). Creative cognition and the brain: Dissociations between frontal, parietal-temporal and basal ganglia groups. Brain Research, 1482, 55-70.

ACTInc. (2014). NationalRanksforTestScoresandCompositeScore. Available online at:http://www.actstudent.org/scores/norms1.html (AccessedAugust3, 2014).

Ansburg, P., \& Hill, K. (2003). Creative and analytic thinkers differ in their use of attentional resources. Personality and Individual Differences, 7, 1141-1152.

Beaty, R. E., \& Silvia, P. J. (2012). Why do ideas get more creative across time? An executive interpretation of the serial order effect in divergent thinking tasks. Psychology of Aesthetics, Creativity, and the Arts, 6, 309-319.

Beaty, R. E., Silvia, P. J., Nusbaum, E. C., Jauk, E., \& Benedek, M. (2014). The roles of associative and executive processes in creative cognition. Memory \& Cognition, 42, 1186-1197.

Benedek, M., Jauk, E., Fink, A., Koschutnig, K., Reishofer, G., Ebner, F., \& Neubauer, A. C. (2014). To create or to recall? Neural 
mechanisms underlying the generation of creative new ideas. NeuroImage, 88, 125-133.

Benedek, M., Könen, T., \& Neubauer, A. C. (2012). Associative abilities underlying creativity. Psychology of Aesthetics, Creativity, and the Arts, 6, 273-281.

Bultitude, J. H., Rafal, R. D., \& List, A. (2009). Prism adaptation reverses the local processing bias in patients with right temporo-parietal junction lesions. Brain: A Journal of Neurology, 132, 1669-1677.

Carson, S. H., Peterson, J. B., \& Higgins, D. M. (2003). Decreased latent inhibition is associated with increased creative achievement in highfunctioning individuals. Journal of Personality and Social Psychology, 85, 499-506.

Carson, S. H., Peterson, J. B., \& Higgins, D. M. (2005). Reliability, validity, and factor structure of the Creative Achievement Questionnaire. Creativity Research Journal, 17, 37-50.

Chrysikou, E. G., \& Thompson-Schill, S. L. (2011). Dissociable brain states linked to common and creative object use. Human Brain Mapping, 32, 665-675.

CollegeBoard. (2012). 2012 College-Bound Seniors Total Group Profile Report New York: The College Board. Available online at: http:// media.collegeboard.com/digitalServices/pdf/research/TotalGroup2012.pdf (AccessedAugust3, 2014).

Coyle, T. R., \& Pillow, D. R. (2008). SAT and ACT predict college GPA after removing $g$. Intelligence, 36, 719-729.

Dreisbach, G., \& Goschke, T. (2004). How positive affect modulates cognitive control: Reduced perseveration at the cost of increased distractibility. Journal of Experimental Psychology: Learning, Memory, and Cognition, 30, 343-353.

Eriksen, C. W., \& Yeh, Y.-Y. (1985). Allocation of attention in the visual field. Journal of Experimental Psychology: Human Perception and Performance, 11, 583-597.

Fink, A., \& Benedek, M. (2014). EEG alpha power and creative ideation. Neuroscience and Biobehavioral Reviews, 44, 111-123.

Fink, A, Grabner, R. H., Benedek, M., Reishofer, G., Hauswirth, V., Fally, M., ... Neubauer, A. C. (2009). The creative brain: Investigation of brain activity during creative problem solving by means of EEG and fMRI. Human Brain Mapping, 30, 734-748.

Fredrickson, B. L., \& Branigan, C. (2005). Positive emotions broaden the scope of attention and thought-action repertoires. Cognition \& Emotion, 19, 313-332.

Fukuda, K., \& Vogel, E. K. (2011). Individual differences in recovery time from attentional capture. Psychological Science, 22, 361-368.

Gasper, K., \& Clore, G. L. (2002). Attending to the big picture: Mood and global versus local processing of visual information. Psychological Science, 13, 34-40.

Goff, K., \& Torrance, E. P. (2002). Abbreviated Torrance Test for Adults manual. Bensenvill: Scholastic Testing Service.

Hedden, T., \& Gabrieli, J. D. E. (2011). Shared and selective neural correlates of inhibition, facilitation, and shifting processes during executive control. Neuroimage, 51, 421-431.

Jonides, J. (1980). Towards a model of the mind's eye's movement. Canadian Journal of Psychology, 34, 103-112.

Jonides, J. (1983). Further towards a model of the mind's eye's movement. Bulletin of the Psychonomic Society, 21, 247-250.

Lamb, M. R., \& Yund, W. (2000). The role of spatial frequency in cued shifts of attention between global and local forms. Perception and Psychophysics, 62, 753-761.

Lee, C. S., \& Therriault, D. J. (2013). The cognitive underpinnings of creative thought: A latent variable analysis exploring the roles of intelligence and working memory in three creative thinking processes. Intelligence, 41, 306-320.

Madden, D. J. (1992). Selective attention and visual search: revision of an allocation model and application to age differences. Journal of Experimental Psychology: Human Perception and Performance, $18,821-836$
Mednick, S. A. (1962). The associative basis of the creative process. Psychological Review, 69, 220-232.

Mendelsohn, G., \& Griswold, B. (1964). Differential use of incidental stimuli in problem solving as a function of creativity. Journal of Abnormal and Social Psychology, 68, 431-436.

Navon, D. (1977). Forest before trees: The precedence of global features in visual perception. Cognitive Psychology, 9, 353-383.

Neçka, E. (1999). Creativity and attention. Polish Psychological Bulletin, $30,85-98$.

Nusbaum, E. C., \& Silvia, P. J. (2011). Are intelligence and creativity really so different? Fluid intelligence, executive processes, and strategy use in divergent thinking. Intelligence, 39, 36-45.

Posner, M. I. (1980). Orienting of attention. Quarterly Journal of Experimental Psychology, 32, 3-25.

Rawlings, D. (1985). Psychoticism, creativity, and dichotic listening. Personality and Individual Differences, 6, 737-742.

Rowe, G., Hirsh, J. B., \& Anderson, A. K. (2007). Positive affect increases the breadth of attentional selection. Proceeding of the National Academy of Sciences of the United States of America, 104, 383-388.

Runco, M. A., \& Acar, S. (2012). Divergent thinking as an indicator of creative achievement. Creativity Research Journal, 24, 66-75.

Russell, J. (1976). Utilization of irrelevant information by high and low creatives. Psychological Reports, 39, 105-106.

Silvia, P. J., Beaty, R. E., Nusbaum, E. C., Eddington, K. M., \& Kwapil, T. R. (2014). Creative motivation: Creative achievement predicts cardiac autonomic markers of effort during divergent thinking. Biological Psychology, 102, 30-37.

Silvia, P. J., Beaty, R. E., \& Nusbaum, E. C. (2013). Verbal fluency and creativity: General and specific contributions of broad retrieval ability (Gr) factors to divergent thinking. Intelligence, 41, 328-340.

Süß, H. M., Oberauer, K., Wittman, W. W., Wilhelm, O., \& Schulze, R. (2002). Working-memory capacity explains reasoning ability - and a little bit more. Intelligence, 30, 261-288.

Torrance, E. P. (1969). Prediction of adult creative achievement among high school seniors. Gifted Child Quarterly, 12, 195-199.

Torrance, E. P. (1974). The Torrance Tests of Creative Thinking-NormsTechnical manual research edition, figural tests, Forms $A$ and $B$. Princeton: Personnel Press.

Vartanian, O. (2009). Variable attention facilitates creative problem solving. Psychology of Aesthetics, Creativity, and the Arts, 3, 57-59.

Vartanian, O., Martindale, C., \& Kwiatkowski, J. (2007). Creative potential, attention, and speed of information processing. Personality and Individual Differences, 43, 1470-1480.

Wallach, M. A., \& Kogan, N. (1965). Modes of thinking in young children: A study of the creativity-intelligence distinction. New York: Holt, Rinehart, \& Winston.

Wegbreit, E., Suzuki, S., Grabowecky, M., Kounios, J., \& Beeman, M. (2012). Visual attention modulates insight versus analytic solving of verbal problems. Journal of Problem Solving, 4, 94-115.

Zabelina, D. L., \& Beeman, M. (2013). Short-term attentional perseveration associated with real-life creative achievement. Frontiers in Psychology, 4, 1-8.

Zabelina, D. L., Condon, D., \& Beeman, M. (2014). Do dimensional psychopathology measures relate to divergent thinking or creative achievement? Frontiers in Psychology, 5, 1-11.

Zabelina, D. L., O'Leary, D., Pornpattananangkul, N., Nusslock, R., \& Beeman, M. (2015). Creativity and sensory gating indexed by the P50: Selective versus leaky attention in divergent thinkers and creative achievers. Neuropsychologia, 69, 77-84.

Zabelina, D. L., \& Robinson, M. D. (2010). Creativity as flexible cognitive control. Psychology of Aesthetics, Creativity, and the Arts, 4, 136. 